\title{
AVALIAÇÃO DE TECNOLOGIAS NO CENTRO CIRÚRGICO, RECUPERAÇÃO PÓS-ANESTÉSICA E CENTRO DE MATERIAL E ESTERILIZAÇÃO
}

\author{
Technology assessment in the surgical center, post-anesthetic \\ recovery, and central sterile supply department \\ Evaluación de la tecnología en el centro quirúrgico, \\ recuperación pos anestésica y centro de material y esterilización \\ Eliane Molina Psaltikidis ${ }^{1}$
}

RESUMO: Introdução: As tecnologias em saúde são essenciais no centro cirúrgico (CC), na recuperação pós-anestésica (RPA) e no centro de material e esterilização (CME). Por isso, há grande pressão para sua incorporação tecnológica, o que demanda alto investimento e elevados custos operacionais. Objetivos: Refletir sobre os conceitos e princípios da avaliação de tecnologias em saúde (ATS) e discutir exemplos de sua aplicação no contexto do CC, da RPA e do CME. Resultados: A metodologia de ATS permite análise dos impactos clínicos, sociais e econômicos da incorporação de tecnologias, buscando melhorar a qualidade de atendimento e a saúde da população. O Ministério da Saúde tem patrocinado diversas iniciativas para difusão dos princípios de ATS que visam subsidiar os gestores para a tomada de decisão em incorporação tecnológica, tanto no âmbito do sistema de saúde quanto nas instituições hospitalares. Conclusão: A equipe de enfermagem deve, na tomada de decisões, apropriar-se da metodologia de ATS para análise crítica do real benefício das tecnologias do bloco operatório. Palavras-chave: Avaliação da tecnologia biomédica. Centros cirúrgicos. Esterilização. Período de recuperação da anestesia.

ABSTRACT: Introduction: Health technologies are essential in the surgical center (SC), in post-anesthetic recovery (PAR), and in the Central Sterile Supply Department (CSSD). Therefore, there is great pressure for the incorporation of technology in them, which demands high investment and high operating costs. Objectives: To propose a reflection on the concepts and principles of the health technology assessment (HTA) and to discuss examples of its application in the context of SC, PAR, and CSSD. Results: The HTA methodology allows analysis of clinical, social, and economic impacts of the incorporation of technologies, seeking to improve the quality of care and the health of the population. The Brazilian Ministry of Health has sponsored several initiatives to disseminate the principles of HTA that seek to support managers' decision-making process regarding technological resources, both within the public health system and in private hospitals. Conclusion: The nursing staff must, during the decision-making process, take ownership of the HTA methodology for critical analysis of the real benefit of the surgical center technologies. Keywords: Technology assessment, biomedical. Surgicenters. Sterilization. Anesthesia recovery period.

RESUMEN: Introducción: Las tecnologías de la salud son esenciales en el centro quirúrgico (CQ), en la recuperación post-anestésica (RPA) y en el centro de material y esterilización (CME). Por lo tanto, existe una gran presión para la incorporación de tecnología en ellos, lo que exige una alta inversión y altos costos de operación. Objetivos: Proponer una reflexión sobre los conceptos y principios de evaluación de las tecnologías de salud (ETS) y discutir ejemplos de su aplicación en el contexto de SC, PAR y MSC. Resultados: La metodología ETS permite analizar los impactos clínicos, sociales y económicos de la incorporación de tecnologías, buscando mejorar la calidad de la atención y la salud de la población. El Ministerio de Salud de Brasil ha patrocinado varias iniciativas para difundir los principios de la ETS que buscan apoyar el proceso de toma de decisiones de los gestores con respecto a los recursos tecnológicos, tanto dentro del sistema público de salud como en los hospitales privados. Conclusión: Durante el proceso de toma de decisiones, el personal de enfermería debe apropiarse de la metodología ETS para el análisis crítico del beneficio real de las tecnologías del centro quirúrgico. Palabras clave: Evaluación de la tecnología biomédica. Centros Quirúrgicos. Esterilización. Período de recuperación de la anestesia.

'Enfermeira; Mestre pelo Programa de Pós-graduação em Saúde do Adulto da Escola de Enfermagem da Universidade de São Paulo (USP); Doutoranda em Clínica Médica da Faculdade de Ciências Médicas da Universidade de Campinas (UNICAMP). Atua como assessora no Núcleo de Avaliação de Tecnologias em Saúde (NATS) do Hospital de Clínicas da UNICAMP - Campinas (SP), Brasil.

Rua Vital Brasil, 251 - Cidade Universitária Zeferino Vaz - CEP: 13083-888 - Campinas (SP), Brasil.

Recebido: 01 set. 2016 - Aprovado: 27 set. 2016

DOI: $10.5327 / Z 1414-4425201600040009$ 


\section{INTRODUÇÃo}

O centro cirúrgico (CC) caracteriza-se como uma unidade hospitalar com uso intenso de tecnologias em saúde e destacada vocação para o pioneirismo na adoção de novas técnicas, equipamentos e produtos para a saúde ${ }^{1}$. Também consiste em uma das áreas de maior custo e faturamento hospitalar. Por tais motivos, recebe grande pressão para a incorporação de novas tecnologias, exercida pelos fabricantes, pelos profissionais de saúde e até pelos próprios pacientes que desejam ter acesso aos procedimentos inovadores em sua assistência.

Cabe salientar que, inevitavelmente, as tecnologias adotadas no CC causam repercussão nos processos de trabalho da recuperação pós-anestésica (RPA) e no centro de material e esterilização (CME). Neste, os impactos são decorrentes de novos equipamentos e instrumentais, em sua maioria de estrutura complexa, que necessitam ser apropriadamente processados. Outra repercussão da aplicação de novas tecnologias é a pressão pela prática do reuso dos produtos para saúde de alto custo, cujos fabricantes recomendam uso único.

Entretanto nem sempre há evidências sólidas da eficácia, efetividade e eficiência dessas novas tecnologias em saúde. Por isso, devem ser ponderados os seus benefícios, riscos e custos. A avaliação de tecnologias em saúde (ATS) consiste em uma metodologia que produz subsídio técnico ao gestor para tomada de decisão, de forma racional e transparente, quanto à incorporação de determinada tecnologia ${ }^{2-4}$.

O presente artigo teve por objetivos trazer uma reflexão sobre os conceitos e princípios da ATS e discutir exemplos de sua aplicação no contexto do CC, da RPA e do CME.

\section{CONCEITOS E PRINCÍPIOS IMPORTANTES SOBRE A AVALIAÇÃO DE TECNOLOGIAS EM SAÚDE}

As tecnologias em saúde englobam medicamentos, equipamentos e procedimentos técnicos, sistemas organizacionais, informacionais, educacionais e de suporte, programas e protocolos assistenciais, por meio dos quais a atenção e os cuidados com a saúde são prestados à população4.

A rápida inovação das tecnologias em saúde e seu impacto nos custos assistenciais preocupam os gestores de sistemas tanto públicos quanto privados, pois o cenário mundial da área da saúde tem apresentado oferta praticamente infinita de opções tecnológicas, em oposição a recursos cada vez menores, limitados e finitos. Além disso, verifica-se grande leque de interesses econômicos envolvidos na expectativa de incorporação das tecnologias ${ }^{3,4}$. Muitos desses interesses são legítimos e pautados nas boas e éticas práticas de mercado na área da saúde, entretanto várias denúncias têm sido feitas sobre ações criminosas para a incorporação de medicamentos e procedimentos de alto custo ou sem benefício algum aos pacientes ${ }^{5-7}$.

A equipe de enfermagem tem contato intenso com as tecnologias em saúde, mesmo com aquelas em que a definição da assistência adotada ao paciente não parte do enfermeiro ${ }^{1}$. Por essa proximidade com as tecnologias, a enfermagem consegue perceber as dificuldades no uso, os problemas em sua aplicação que possam oferecer risco aos pacientes e à equipe, a reação do paciente diante da tecnologia aplicada e as necessidades não atendidas pelas tecnologias atuais. Além desse papel, muitas vezes o enfermeiro pode atuar como gestor, tomador de decisões e formador de opinião quanto à incorporação tecnológica.

Todos os gestores em saúde necessitam de informações confiáveis e detalhadas que lhes permitam tomar decisões racionais, coerentes e transparentes no momento de estabelecer prioridades na incorporação de tecnologias, tendo como meta obter o máximo benefício com o orçamento disponível. A ATS é a principal ferramenta metodológica para esse processo, pois analisa os impactos clínicos, sociais e econômicos da incorporação das tecnologias, visando melhorar a qualidade de atendimento e a saúde da população $0^{3,4,8}$.

A ATS possibilita mensurar a eficácia (comprovação de resultados favoráveis para a condição de saúde a que se destina), a efetividade (confirmação de que os resultados favoráveis identificados nas pesquisas de eficácia são mantidos na prática assistencial) e a eficiência (análise dos benefícios nos desfechos no tocante aos custos) das tecnologias, em todas as fases do seu ciclo de vida. A ATS também pode gerar estudos de monitoramento de horizonte tecnológico para as tecnologias inovadoras, estudos de custo-efetividade e efetividade comparativa para as tecnologias em difusão e estudos de obsolescência e desincorporação para as que já estão em fase de descarte $e^{2-4,8}$.

Para a realização da ATS, alguns princípios metodológicos são fundamentais:

- pergunta da análise explícita e baseada na ferramenta PICO, que define a população $(\mathrm{P})$ a que se destina, a intervenção (I), ou seja, a tecnologia analisada, o comparador $(\mathrm{C})$ e os desfechos relevantes $(\mathrm{O}$, de outcomes) a serem adotados;

- busca ampla, sistemática e reprodutível da literatura, nas principais bases eletrônicas, agências de ATS e literatura cinzenta, preferencialmente sem restrição de idioma de publicação; 
- análise dos estudos por no mínimo dois revisores independentes e sem conflito de interesses com a tecnologia avaliada;

- seleção dos estudos pela melhor evidência disponível, priorizando aqueles com desenho de menor risco de viés;

- avaliação da qualidade metodológica dos estudos por instrumentos validados;

- análise da qualidade do corpo de evidências para cada desfecho definido no PICO;

- análise crítica dos resultados perante a realidade de saúde local e seus impactos clínicos e econômicos;

- avaliação econômica e estudos de impacto orçamentário pelas metodologias da economia da saúde;

- elaboração do relatório da ATS na linguagem e perspectiva do gestor demandante ${ }^{3,9,10}$.

\section{AVALIACÃO DE TECNOLOGIAS EM SAUÚDE NO BRASIL}

Apesar de os princípios de avaliação de tecnologias em saúde já estarem firmados em muitos países, no Brasil esse tema ainda é novo. O Ministério da Saúde tem investido na estruturação de Núcleos de Avaliação de Tecnologia em Saúde (NATS) nos hospitais de ensino, secretarias de saúde, instituições de pesquisa e grandes hospitais do país. Tais núcleos auxiliam na capacitação de profissionais da área, assessoram os gestores da instituição nas decisões sobre incorporação tecnológica e atendem a demandas do Ministério da Saúde e das secretarias com estudos de ATS para análise de incorporação no Sistema Único de Saúde (SUS). Os NATS agregam-se à Rede Brasileira de ATS (REBRATS), também vinculada ao Ministério da Saúde, que propicia interação, cursos de capacitação, organização de grupos de trabalho e elaboração de diretrizes metodológicas que balizam e uniformizam os documentos de ATS produzidos no país (http: / / rebrats.saude.gov.br/).

O principal marco legal para a institucionalização da ATS no Brasil deu-se pela Lei . $^{\circ} 12.401$, de 2011 , que alterou a Lei n. ${ }^{\circ} 8.080$, de 1990 , que rege o SUS, especificamente no artigo $19^{11}$. A nova redação desse artigo delimita a assistência terapêutica integral garantida pelo SUS, que passa a ser a estabelecida por diretrizes terapêuticas e protocolos clínicos nacionais ou mediante evidências científicas de eficácia, segurança, efetividade e custo-efetividade para as diferentes fases evolutivas da doença ou do agravo à saúde. Ou seja, qualquer procedimento, medicamento ou produto para saúde fará parte da assistência integral do SUS, desde que haja avaliação de tecnologia que justifique sua incorporação, mediante seus benefícios, em âmbito nacional. Essa lei também instituiu a Comissão Nacional de Incorporação de Tecnologias no SUS (CONITEC) para assessorar o Ministério da Saúde na incorporação, exclusão ou alteração das tecnologias em saúde no sistema público, além de elaborar e atualizar os protocolos clínicos e as diretrizes terapêuticas nacionais (http:/ / conitec.gov.br/).

Desde a sua instauração, em 2012, até julho de 2016, a CONITEC já avaliou 492 demandas, e 56\% delas foram encaminhadas pelo próprio Ministério da Saúde visando à atualização do arsenal terapêutico e diagnóstico no SUS. Do total de demandas, a maioria foi de medicamentos (65\%), seguida de procedimentos (21\%) e de produtos para saúde (14\%). Por meio dessa atuação, a CONITEC possibilitou a incorporação de 173 tecnologias novas no rol do SUS, com impacto orçamentário estimado em $\mathrm{R} \$ 2,5$ bilhões $^{12}$.

A atuação da CONITEC tem impacto ainda no sistema de saúde privado, pois, com a incorporação da tecnologia no SUS (por intermédio de evidências de efetividade), as operadoras de planos de saúde se veem pressionadas a também ampliarem sua cobertura.

Apesar dessa evolução, os princípios de ATS são pouco praticados pelos gestores dos serviços de saúde locais, em grande parte em razão da falta de informações sobre o recurso para tomada de decisão e pela carência de profissionais capacitados a elaborar avaliações para as suas demandas institucionais. Diante da essa realidade, o Ministério da Saúde tem apoiado diversos cursos sobre ATS para gestores e estimulado a ampliação do número de NATS pelo país.

\section{REFLEXÕES SOBRE A APLICACÃO DE AVALIAÇ̃̃̃O DE TECNOLOGIAS EM SAỨDE EM CENTRO CIRÚRGICO, RECUPERACÃOO PÓS-ANESTÉSICA E CENTRO DE MATERIÁL E ESTERILLZAÇÃ̃o}

Há diversos questionamentos sobre o uso excessivo de tecnologias na área cirúrgica e seus impactos nos custos da assistência à saúde, sem os correspondentes benefícios ao paciente. A revista Época, em maio de 2015, ao publicar ampla reportagem acerca dos custos da saúde, citou que os médicos do Hospital Israelita Albert Einstein, em São Paulo, SP, reavaliaram a condição de quase 1.500 clientes da seguradora Bradesco Saúde que estavam prestes a fazer uma cirurgia de coluna. Como conclusão, verificaram que dois terços deles não precisariam do procedimento e que teriam melhor indicação para tratamento conservador ${ }^{13}$. Tal realidade não é exclusiva do Brasil. 
Em agosto de 2016, o The New York Times, em reportagem intitulada "Why 'useless' surgery is still popular", questionou a realização rotineira de cirurgias ortopédicas que estudos com alta qualidade de evidência já demonstraram que não apresentam benefício em relação ao tratamento conservador ${ }^{14-17}$. Essas questões precisam permear toda a sociedade para que os profissionais e usuários do sistema de saúde se tornem mais críticos quanto às práticas da assistência à saúde.

Algumas técnicas cirúrgicas muito valorizadas e recomendadas, ao serem examinadas sob sistemática ATS, mostram-se apoiadas em pesquisas de baixa qualidade metodológica, ou seja, com carência de evidências dos seus reais benefícios. Nessa situação, pode-se citar o esfíncter urinário artificial, que, apesar de ser considerado padrão ouro para tratamento de incontinência urinária moderada ou grave pós-prostatectomia radial, se sustenta em apenas um estudo controlado randomizado, com pequeno número amostral e baixa qualidade metodológica, comparado apenas à injeção de macroplastique. Os outros estudos sobre o esfíncter urinário artificial são observacionais de muito baixa qualidade que apontaram resultados significativos na continência e satisfação do paciente, porém com maior risco de complicações (infecção, estenose uretral, mau funcionamento, necessidade de revisão do dispositivo ao longo dos anos e eventual troca ou retirada) $)^{18,19}$.

A cirurgia robótica é outro exemplo de tecnologia de alto custo, com muita repercussão nos eventos científicos e na mídia, que ainda não tem, porém, evidência sólida sobre benefícios que justifiquem sua incorporação na prática assistencial. No Brasil, foi realizada uma investigação, por demanda do Ministério da Saúde, sobre prostatectomia radical assistida roboticamente (PRAR) comparada à técnica aberta e à laparoscópica ${ }^{20}$. O trabalho foi desenvolvido em três hospitais que já tinham o robô cirúrgico e faziam cerca de $25 \mathrm{PRAR} / \mathrm{mês}$. Os resultados indicaram menor perda de sangue na PRAR em relação à cirurgia aberta, mas, comparando-se à técnica laparoscópica, a diferença não foi relevante. Os outros desfechos analisados, tais como tempo de internação e de cirurgia, não foram animadores, entretanto os custos de aquisição do equipamento e de insumos foram enormes. O primeiro estudo clínico randomizado sobre PRAR está em andamento; os resultados parciais foram recentemente publicados com o acompanhamento dos pacientes por 12 semanas $^{21}$. Nele, houve diferença significativa entre o grupo de PRAR, em comparação à cirurgia aberta, apenas em dor nas primeiras 24 horas e na primeira semana de pós -operatório, em perda de sangue e em tempo de internação.
Todavia, não houve diferença significativa na hemotransfusão, e a diferença no tempo de sala não foi relevante. Entretanto o resultado mais surpreendente é que não se constatou diferença significativa entre os grupos para os desfechos funcionais como a função urinária, a função sexual, a margem positiva nas amostras cirúrgicas e o tempo para retorno ao trabalho. A conclusão dos autores é que existe necessidade de mais acompanhamento e que, para prostatectomia radical, a experiência do cirurgião é mais importante do que o tipo de abordagem cirúrgica.

Quanto aos instrumentos utilizados nas cirurgias, o NATS do Hospital de Clínicas da Universidade Estadual de Campinas (UNICAMP) teve a oportunidade de avaliar os grampeadores cirúrgicos de uso único, por demanda da instituição, por conta do alto custo dos produtos e das restrições de ressarcimento pelo SUS, que prevê seu uso somente para algumas cirurgias. $\mathrm{Na}$ análise da literatura, com estudos de alta qualidade de evidência, em cirurgias do trato gastrointestinal e pulmonar, não houve evidência de melhores desfechos clínicos pós-operatórios com o uso dos grampeadores. Como a análise do seu consumo na instituição resultou em custo $25 \%$ acima do ressarcido, o hospital optou por restringir sua utilização apenas para os procedimentos nos quais os grampeadores são previstos pelo SUS ${ }^{22}$.

Outra tecnologia que tem sido bastante divulgada é o sistema de desinfecção de superfícies no-touch com vaporização de peróxido de hidrogênio ou radiação ultravioleta. Esses aparelhos são indicados para limpeza terminal de áreas críticas, especialmente nas quais há risco de contaminação com bactérias multirresistentes e Clostridium difficile. Embora os estudos apresentem eficácia de tais sistemas na inativação de vasto espectro de microrganismos e algum resultado na redução de infecções relacionadas, especialmente em situações de surto, a operacionalização desse método é o grande fator limitante. Ou seja, a efetividade deixa a desejar, pois há necessidade de limpeza prévia de todas as superfícies da área, vedação de entrada e saída de ar, bloqueio da área durante o tempo de aplicação e de exaustão (que pode levar mais de 1 hora), treinamento da equipe, além dos custos do equipamento e insumos. No caso da radiação ultravioleta, tem-se ainda a limitação do sombreamento, pois onde a luz não atingir não haverá a ação microbicida. Um estudo conduzido pela Agência Canadense de ATS (CADTH) analisou esse sistema e concluiu que não há evidências suficientes para recomendar a sua incorporação ${ }^{23}$.

No que se refere ao CME, vê-se grande carência de estudos de ATS, apesar da vasta gama de novos produtos para a área. Uma agência de ATS da província de Quebec, no Canadá, fez 
uma avaliação comparativa das pasteurizadoras e das lavadoras termodesinfetadoras para material de assistência respiratória, comprovando o custo-efetividade de ambas, com pequena vantagem às lavadoras, por possibilitarem limpeza em diferentes ciclos ${ }^{24}$.

No Hospital de Clínicas da UNICAMP, em decorrência dos questionamentos perante a substituição do glutaraldeído por ácido peracético na desinfecção de endoscópios, foi realizada ATS sobre os desinfetantes de alto nível. A análise sumarizou as evidências sobre o tema no tocante à eficácia, à compatibilidade e a limitações de cada germicida, bem como demonstrou a carência mundial de estudos a respeito de danos aos equipamentos relacionados aos diferentes desinfetantes ${ }^{25}$.

\section{CONSIDERAÇÕES FINAIS}

A inevitável escassez de recursos na área da saúde e a pressão por incorporação tecnológica têm levado à disseminação dos princípios de ATS entre os gestores, em todos os níveis do sistema de saúde. Há muito o que expandir na avaliação de tecnologias em saúde adotadas em CC, RPA e CME, o que abre grande espaço para os profissionais de enfermagem que se capacitem nas ferramentas metodológicas de ATS. A tomada de decisão para investimentos nessas áreas envolve, muitas vezes, relevante aporte financeiro e exige análises pautadas na melhor evidência disponível, visando garantir que a razão entre custo e efetividade seja favorável.

\section{REFERÊNCIAS}

1. Associação Brasileira de Enfermeiros de Centro Cirúrgico, Recuperação Anestésica e Centro de Material e Esterilização (Sobecc). Práticas recomendadas Sobecc. São Paulo: Manole; 2013.

2. Brasil. Ministério da Saúde. Secretaria de Ciência, Tecnologia e Insumos Estratégicos. Glossário temático: economia da saúde. Brasília: Ministério da Saúde; 2005. 60 p.

3. Brasil. Ministério da Saúde. Secretaria de Ciência, Tecnologia e Insumos Estratégicos. Diretrizes metodológicas: elaboração de pareceres técnico-cientíícos. $4^{\mathrm{a}}$ ed. Brasília: Ministério da Saúde; 2014. 80 p.

4. Brasil. Ministério da Saúde. Secretaria de Ciência, Tecnologia e Insumos Estratégicos. Política Nacional de Gestão de Tecnologias em Saúde. Brasília: Ministério da Saúde; 2010. 48 p.

5. Segatto C. Os falsos doentes de $\mathrm{R} \$ 9,5$ milhões: os bastidores de uma das maiores fraudes já descobertas no Brasil envolvendo ações judiciais para fornecimento de remédios de alto custo. Revista Época [online]. 2016 jun. 22 [citado 2016 jul. 21]. Disponível em: http:// epoca.globo.com/vida/noticia/2016/06/os-falsos-doentes-de-r-95milhoes.html

6. Carmo SGD, Amâncio T. Investigação aponta fraude em compra de material para cirurgia no HC. Folha de S. Paulo [online]. 2016 jul. 18 [citado 2016 ago. 29]. Disponivel em: http://www1.folha.uol.com.br/ cotidiano/2016/07/1792811-investigacao-aponta-fraude-de-r-135mi-no-tratamento-do-mal-de-parkinson.shtml

7. Pivetta M. A prova final da fosfoetanolamina: testes clínicos em seres humanos devem atestar se o composto pode ser útil no tratamento de algum tipo de câncer. Revista Pesquisa FAPESP [online]. 2016 [citado 2016 jul. 11];243:16-23. Disponível em: http://revistapesquisa. fapesp.br/2016/05/17/a-prova-final-da-fosfoetanolamina/

8. Toma TS, Soares AC, Bortoli MC, Pirotta KCM, Venâncio SI, Derbli M, editors. Avaliação de tecnologias e inovação em saúde no SUS: desafios e propostas para a gestão. São Paulo: Instituto de Saúde [online]; 2015 [citado 2016 ago. 02]. p. 13-100. Disponível em: http:// www.saude.sp.gov.br/resources/instituto-de-saude/homepage/ temas-saude-coletiva/pdfs/ats_inova_saude_capa_miolo.pdf
9. Brasil. Ministério da Saúde. Secretaria de Ciência, Tecnologia e Insumos Estratégicos. Diretrizes metodológicas: elaboração de revisão sistemática e metanálise de ensaios clínicos randomizados. Brasília: Ministério da Saúde [online]; 2012 [citado 2016 maio 30]. 92 p. Disponível em: http://rebrats.saude.gov.br/diretrizes-metodologicas

10. Brasil. Ministério da Saúde. Secretaria de Ciência, Tecnologia e Insumos Estratégicos. Diretrizes metodológicas: estudos de avaliação econômica de tecnologias em saúde. 2a ed. Brasília: Ministério da Saúde [online]; 2014 [citado 2016 jun. 04]. 132 p. Disponível em: http://rebrats.saude.gov.br/diretrizes-metodologicas

11. Brasil. Lei n. ${ }^{\circ} 12.401$, de 28 de abril de 2011 . Altera a Lei $n .{ }^{\circ} 8.080$, de 19 de setembro de 1990, para dispor sobre a assistência terapêutica e a incorporação de tecnologia em saúde no âmbito do Sistema Único de Saúde - SUS. Diário Oficial da União; 29 abr. 2011 [citado 2016 ago. 07]. Disponível em: http://www.planalto.gov.br/ccivil_03/_Ato20112014/2011/Lei/L12401.htm

12. Petramale CA. Conhecendo a CONITEC. Webconferência Conitec em Evidência de 29 de agosto de 2016 [online]. [acesso 2016 set. 04]. Disponível em: https://www.youtube.com/watch?v=TtAXnlb0yZ8

13. Quanto custa a sua saúde? Revista Exame. 2015 maio;1090 [citado 2016 mar. 17]. Disponível em: http://exame.abril.com.br/revista-exame/ edicoes/1090/

14. Kolata G. Why "useless" surgery is still popular. The New York Times [online]. 2016 ago. 03 [citado 2016 ago. 28]. Disponível em: http:// www.nytimes.com/2016/08/04/upshot/the-right-to-know-that-anoperation-is-next-to-useless.html?_r=0

15. Kise NJ, Risberg MA, Stensrud S, Ranstam J, Engebretsen L, Roos EM. Exercise therapy versus arthroscopic partial meniscectomy for degenerative meniscal tear in middle aged patients: randomized controlled trial with two year follow-up. BMJ. 2016;354:i3740. DOI: http://dx.doi.org/10.1136/bmj.i3740

16. Thorlund JB, Juhl CB, Roos EM, Lohmander LS. Arthroscopic surgery for degenerative knee: systematic review and meta-analysis of benefits and harms. BMJ. 2015;350:h2747. DOl: http://dx.doi.org/10.1136/bmj.h2747 
17. Kallmes DF, Comstock BA, Heagerty PJ, Turner JA, Wilson DJ, Diamond TH, et al. A randomized trial of vertebroplasty for osteoporotic spinal fractures. N Engl J Med. 2009;361:569-79. DOI: 10.1056/ NEJMoa0900563

18. Silva LA, Andriolo RB, Atallah AN, da Silva EMK. Surgery for stress urinary incontinence due to presumed sphincter deficiency after prostate surgery. Cochrane Database Syst Rev. 2011;(4):CD008306. DOI: 10.1002/14651858.CD008306.pub2

19. Psaltikidis EM, Bustorff-Silva JM, Resende MR. Efficiency of the artificial urinary sphincter in the treatment of post prostatectomy urinary incontinence. Health Technology Assessment International Annual Meeting [online], Oslo (Noruega), 2015 [citado 2016 mar. 17]. Disponível em: http://pt.slideshare.net/REBRATSoficial/ht-ai-2015poster-238-efficiency-of-the-artificial-urinary-sphincter-49783062

20. Brasil. Ministério da Saúde. Departamento de Ciência e Tecnologia, Secretaria de Ciência, Tecnologia e Insumos Estratégicos. Prostatectomia radical assistida roboticamente. Boletim Brasileiro de Avaliação de Tecnologias em Saúde. 2012;7(20).

21. Yaxley JW, Coughlin GD, Chambers SK, Occhipinti S, Samaratunga $\mathrm{H}$, Zajdlewicz L, et al. Robot-assisted laparoscopic prostatectomy versus open radical retropubic prostatectomy: early outcomes from a randomised controlled phase 3 study. The Lancet [online]. 2016 jul. 26 [citado 2016 jul. 19];388(10049):1057-66. Disponível em: http:// dx.doi.org/10.1016/S0140-6736(16)30592-X

22. Psaltikidis EM, Resende MR, Bustorff-Silva JM. Efficiency analysis of surgical staplers compared to manual suture for open and laparoscopic surgery. Value Health. 2015;18(7):A543. DOI: http:// dx.doi.org/10.1016/j.jval.2015.09.1722

23. Canadian Agency for Drugs and Technologies in Health (CADTH). Nonmanual techniques for room disinfection in healthcare facilities: a review of clinical effectiveness and guidelines. Rapid Response Report. Canadá; 2014 [citado 2016 jun. 23]. Disponível em: https://www.cadth.ca/ about-cadth/what-we-do/products-services/rapid-response-service

24. Agence d'évaluation des technologies et des modes d'intervention en santé (AETMIS). Analyse comparative de la pasteurisation et de la désinfection thermique dans un laveur-désinfecteur: dispositifs d'anesthésie et de soins respiratoires. ETMIS. 2009;5(7).

25. Psaltikidis EM, Leichsenring ML, Nakamura MHY, Bustorff-Silva JM, Passeri LA, Venâncio SI. Desinfetantes de alto nível alternativos ao glutaraldeído para processamento de endoscópios flexíveis. Cogitare Enferm. 2014;19(3):465-74. DOI: http://dx.doi.org/10.5380/ ce.v19i3.35455 